\title{
Fabrication of Mesostructured Silica and Titania Rods on Substrates by Using Polycarbonate Membranes
}

\author{
Norihiro Suzuki ${ }^{1}$ and Yusuke Yamauchi ${ }^{1,2}$ \\ ${ }^{1}$ World Premier International (WPI) Research Center for Materials Nanoarchitectonics (MANA), \\ National Institute for Materials Science (NIMS), Tsukuba, Ibaraki 305-0044, Japan \\ ${ }^{2}$ PRESTO, Japan Science and Technology Agency (JST), Kawaguchi, Saitama 332-0012, Japan
}

Correspondence should be addressed to Yusuke Yamauchi, yamauchi.yusuke@nims.go.jp

Received 30 November 2009; Accepted 11 March 2010

Academic Editor: Yanqiu Zhu

Copyright (c) 2010 N. Suzuki and Y. Yamauchi. This is an open access article distributed under the Creative Commons Attribution License, which permits unrestricted use, distribution, and reproduction in any medium, provided the original work is properly cited.

By using the polycarbonate membrane a template, mesoporous silica rods are fabricated on a silicon substrate in one pot. From scanning electron microscope (SEM) images, the creation of fibrous morphology is confirmed over the entire area. The diameter of the obtained rods is consistent with that of the template. Transmission electron microscope (TEM) images revealed that the tubular mesochannels are uniaxially oriented parallel to the longitudinal axis of the silica rods. The mesoporous titania rods with anatase crystalline frameworks are also fabricated.

\section{Introduction}

Mesoporous/mesostructured one-dimensional (1D) materials (such as rods and tubes) are attractive, because they can be utilized as noble optical, electric and magnetic devices. One of the fabrication methods for the 1D material is the template-directed synthesis. In this process, the precursor solution is introduced into the pores of the template. By adding the surfactant into the precursor solution, the selforganization of surfactant micelles occurs, which derives the ordered mesostructures within the template. After removing the template and surfactants, the mesoporous $1 \mathrm{D}$ materials can be obtained.

The fabrication of the mesoporous 1D materials on a substrate is important for practical application. Recently, Zhu et al. reported the synthesis of mesostructured silica tubes on a silicon substrate using the porous anodic alumina (PAA) template [1]. In this study, though the fabricated tubes were oriented perpendicularly to the substrate, a strong vacuum condition was required to enter the surfactant/silica source into the pores of the PAA template. Furthermore, because the PAA template was removed by the use of phosphoric acid, this fabrication method is not applicable to compositions with low tolerance for acid.
To overcome it, we focus on the polycarbonate (PC) membrane. The PC membrane has several advantages compared to the PAA [2-4]. Firstly, since the PC is stable under acidic condition, the side reactions are prevented. In addition, the PC membrane and the surfactants can be removed simultaneously by calcination. In this paper, we demonstrate the one-pot fabrication of mesoporous silica rods on a silicon substrate using the PC template. Without using the strong vacuum condition, the silica rods were successfully fabricated on the substrate. Our facial fabrication procedure was also applied for the fabrication of mesoporous titania rods.

\section{Experimental}

2.1. Materials. Tetraethyl orthosilicate (TEOS, extra pure reagent) as a silica source was purchased from Nacalai Tesque, Inc. Titanium tetraisopropoxide (as titania source) was obtained from Wako Pure Chemical Industries, Ltd. Ethanol, dilute hydrochloric acid $(\mathrm{pH}=2)$, and concentrated hydrochloric acid $(35.5 \mathrm{w} \%)$ were obtained from Nacalai Tesque, Inc. A triblock copolymer P123 was obtained from Sigma-Aldrich, Inc. Polycarbonate membrane filters with $200 \mathrm{~nm}$ diameter pores were purchased from Advantec Co., Ltd. 

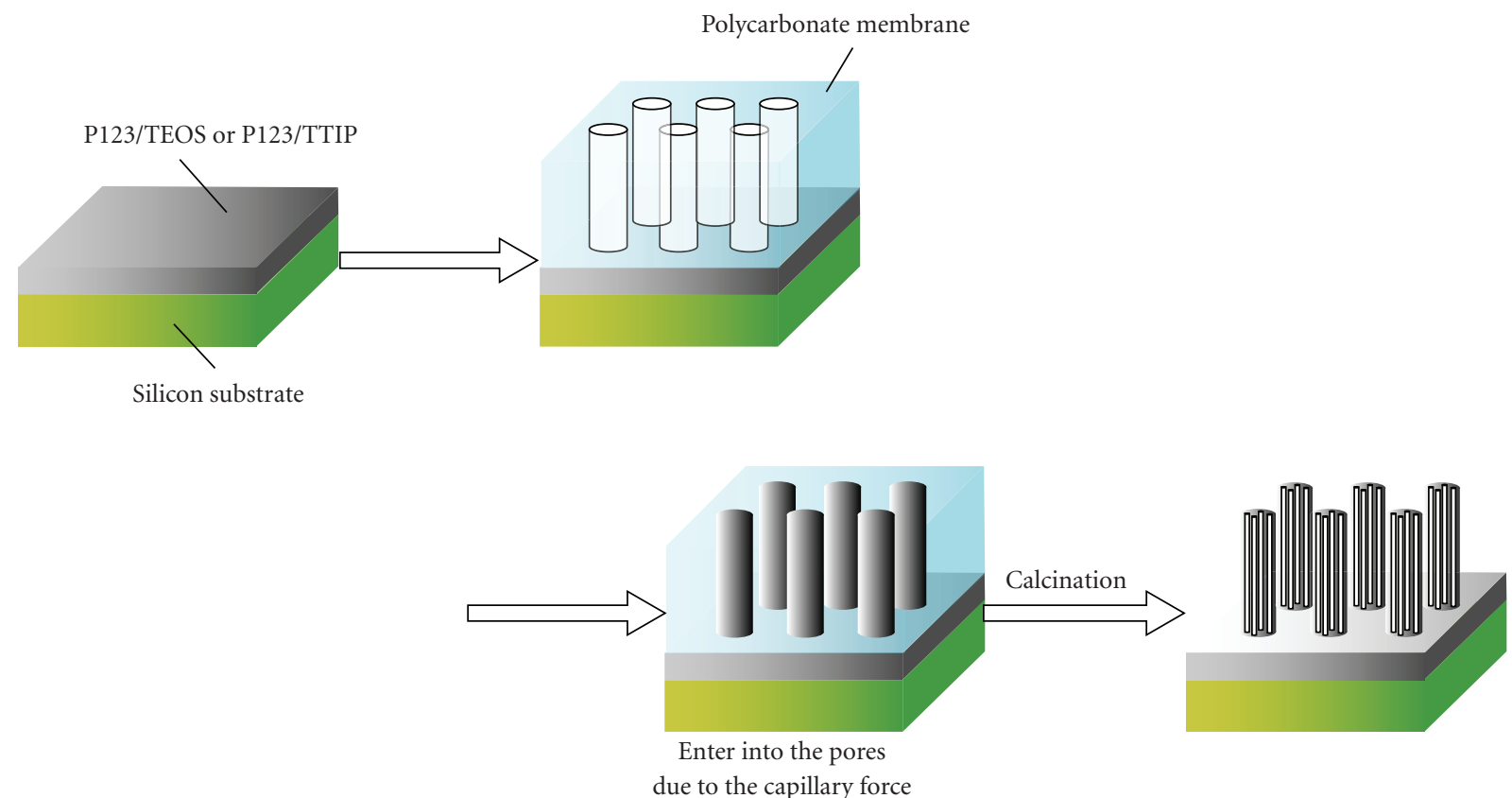

FIGURE 1: Schematic presentation of the fabrication of mesoporous silica and titania rods using the polycarbonate (PC) membrane as the template on a silicon substrate.

\subsection{Preparation of Precursor Solutions. The mixture of} tetraethyl orthosilicate (TEOS, $5.2 \mathrm{~g}$ ), ethanol $(6 \mathrm{~g})$, and dilute aqueous $\mathrm{HCl}(\mathrm{pH}=2,2.7 \mathrm{~g})$ was stirred for 20 minutes. Then triblock copolymer P123 (1.38g) and ethanol (4g) were added and the mixture was stirred for 3 hours [5]. In the preparation of the titania precursor solution, we followed the paper by $\mathrm{Wu}$ et al. [6]. Concentrated $\mathrm{HCl}(35.5 \mathrm{w} \%$, $0.74 \mathrm{~g}$ ) was added into titanium tetraisopropoxide (TTIP, $1.05 \mathrm{~g}$ ) and stirred vigorously for 10 minutes. This solution was added drop by drop into an ethanol solution $(3 \mathrm{~g})$ of P123 (0.2 g), then stirred for 15 minutes.

\subsection{Preparation of Mesoporous Silica and Titania Rods.} The fabrication procedure of mesoporous rods is shown schematically in Figure 1. The thin film was prepared by spin-coating at $8000 \mathrm{rpm}$ (Silica) and $4000 \mathrm{rpm}$ (Titania) on a silicon wafer cleaned with ethanol. After the spincoating, a polycarbonate membrane filter was placed on the film. Because the surface of the spin-coated film was not dried out completely, P123/TEOS and P123/TTIP sources can penetrate into the pores of the PC membrane due to the capillary force. The silica film with the PC membrane was dried in air for 1 day and the titania film was aged at $-20^{\circ} \mathrm{C}$ for 1 day. These as-synthesized films were calcined at $500^{\circ} \mathrm{C}$ for 8 hours (Silica) or $400^{\circ} \mathrm{C}$ for 4 hours (Titania) to remove the PC membrane and surfactants simultaneously.

2.4. Characterization. SEM images were recorded with a Hitachi S-4800 field emission scanning electron microscope. Before the observation, SEM samples were coated with Pt. TEM images were obtained using a JEOL JEM-2010. During the measurement, the accelerating voltage of the electron beam was set to be $200 \mathrm{kV}$.

\section{Results and Discussion}

The SEM image of the silica rods is shown in Figure 2(a). A large number of rods were successfully fabricated over the entire area of the substrate. The estimated diameter of the obtained fiber was about $200 \mathrm{~nm}$, which consisted with the pore size of the PC membranes. These facts indicate that the size of rods can be controlled by changing the pore sizes of the PC membranes. The length of the fabricated fibers ranged between $1 \mu \mathrm{m}$ to $2 \mu \mathrm{m}$, which was much shorter than the PC membrane thickness (about $10 \mu \mathrm{m}$ ). The main reason is that the spin-coated film was so thin that the precursor source cannot fulfill the entire pore space of the PC template. Titania rods were also fabricated as shown in Figure 2(b), revealing that our procedure is applicable beyond silica. The densities of the rods were lower rather than that of the silica tubes/rods prepared by PAA membranes [2, 7-10], because the number of the pores in the PC membranes is relatively lower (Figure 3). Also, the fabricated rods were randomly placed and inclined to the substrate (Figure 2), because the pores in the PC membranes are randomly dispersed and the pores are not oriented completely perpendicular, but are tilted to the substrate (Figure 3).

Almost the P123/TEOS and P123/TTIP sources could penetrate into the pores of the PC membrane due to the capillary force, because the continuous films regions on the $\mathrm{Si}$ substrate were not observed clearly from the cross-sectional SEM images (Figures 2(c) and 2(d)). But, it was revealed that, from a highly magnified SEM image (Figure 4), very thin films (less than $30 \mathrm{~nm}$ ) with uniform mesopores covered the area of the substrate.

Furthermore, the mesostructures inside the silica rods were investigated directly by taking the TEM images perpendicular to the long axis of the fibers (Figure 5). Tubular 


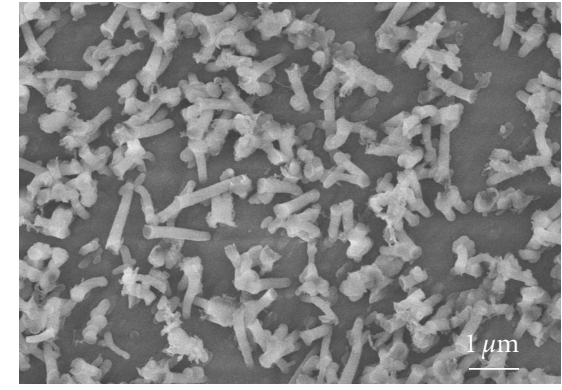

(a) Silica

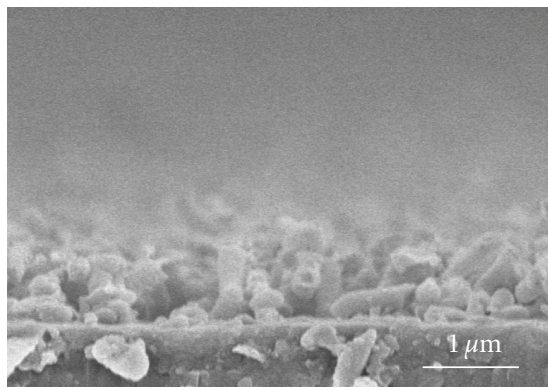

(c) Silica

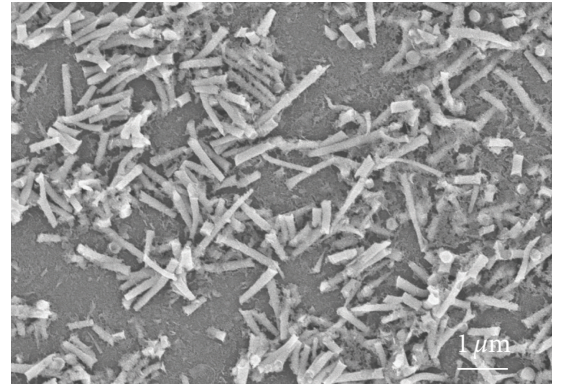

(b) Titania



(d) Titania

Figure 2: SEM images of mesoporous silica (a) and (c) and titania (b) and (d) rods on the silicon substrate: Figures (a) and (b) are topsurface images, while Figures (c) and (d) are cross-sectional images.

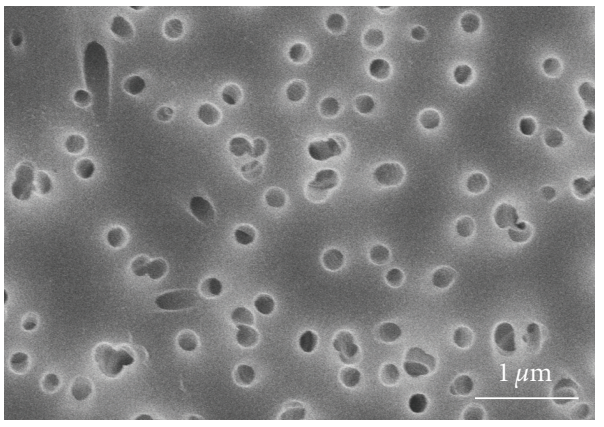

FIGURE 3: SEM image of top-surface of the used PC membranes. Although the pore size is uniform (around $200 \mathrm{~nm}$ ), the pores are randomly distributed on the surface.

mesopores were observed in the rod and these mesochannels were uniaxially oriented parallel to the longitudinal axis of the rod. The pore diameter of mesopores was estimated to be $6.2 \mathrm{~nm}$, which coincided well with the result of $\mathrm{N}_{2}$ gas adsorption-desorption $(6.0 \mathrm{~nm})$ [4]. In the SAXS profiles of silica rods, peaks assignable to (10), (11), and (20) peaks of $2 \mathrm{D}$ hexagonal symmetries ( $p 6 m$ ) were observed (not shown), confirming the mesostructural ordering.

The appearance of the mesopore channels parallel to the longitudinal axis is noteworthy. It has been well known that there are two types of orientations of the mesochannels in a cylindrical confide space. One is "columnar orientation" in which the tubular mesochannels are aligned along the long

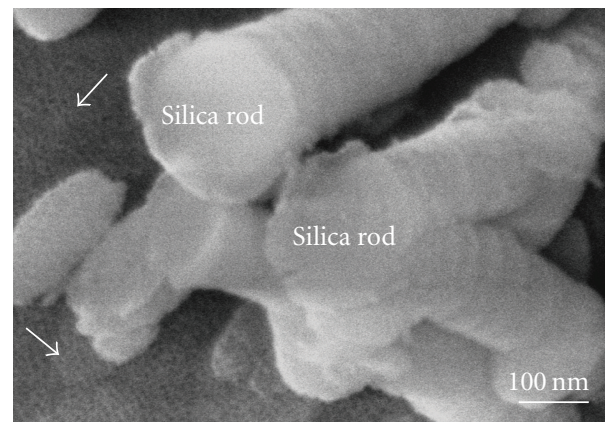

FIGURE 4: High-magnified SEM image. The mesoporous silica rods are deposited on the substrate. The arrows indicate the formation of uniform mesopores on the substrate surface.

axis of the cylinders. The other one is "circular orientation" in which the mesochannels are circularly formed inside the cylinders (i.e., stacked-donuts structures) [7]. In the use of porous anodic alumina (PAA) membranes with P123 surfactants, the "circular orientation" is mainly formed inside the cylindrical channels, even though the mesochannels orientation with respect to the long axis of the cylinders can be tuned according to the silica-to-P123 ratio and the salt content in the precursor solution and the relative humidity during the solvent evaporation [7-9]. On the other hand, in the use of PC membranes with P123 surfactants, the interaction of silica-P123 species with the PC surfaces is weaker than that with the PAA surfaces, and any preferred 


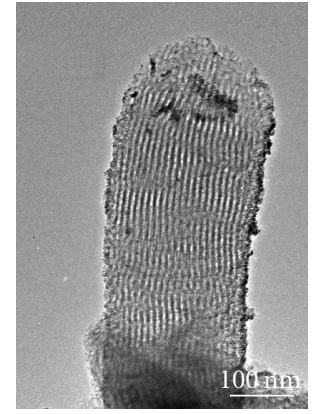

(a)

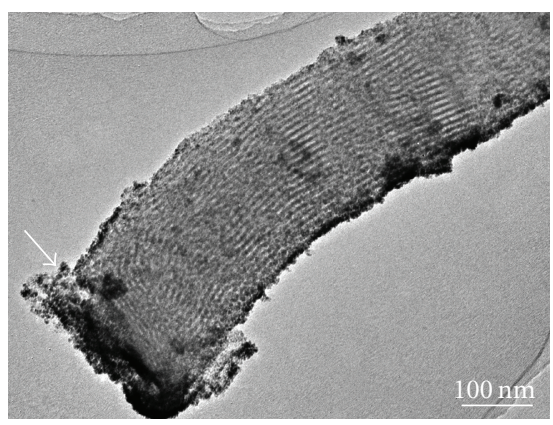

(b)

Figure 5: TEM images of the mesoporous silica rod after the calcination. The arrow in Figure (b) indicates the interface between the silica rod and the Si substrate (i.e., the edge of the mesoporous silica rod).

orientations were not confirmed. In this case, both the "columnar orientation" and the "circular orientation" were observed [4]. In the present system, however, only "columnar orientation" was observed in the rods. When the P123/TEOS source penetrates into the pores of the membrane due to the capillary force (Figure 1), the motion of silica-P123 species in a uniaxial direction to the long axis of the PC channels occurs. At this stage, the tubular mesochannels can be anisotropically arranged along the flow direction (i.e., along the cylinders of the PC membranes), because an interaction between the P123/TEOS sources and the PC surface is weak. At the interface between the silica rod and the Si substrate, it was proved that the mesochannels were oriented parallel to the Si substrate, due to the strong interaction between the silica-P123 species and the Si substrate surface. This phenomenon has been normally observed previously [5].

In the case of the mesoporous titania rods, it is expected that crystallization as well as template removal will occur during the calcination procedure [6]. Disordered mesostructures are observed inside the rods (not shown). In the XRD pattern of titania samples, several diffraction peaks were detected (not shown). These peaks were well matched with the diffraction pattern of anatase titania, revealing that fabricated titania rods have anatase crystalline frameworks. The crystal size of anatase titania was estimated using the Scherrer equation: by using (101) reflection peak, the crystal size was roughly estimated to be around $4.8 \mathrm{~nm}$. Fabrication of mesoporous rods with such crystallized frameworks will be important for a practical application such as catalyst.

\section{Conclusion}

Mesostructured silica and titania rods on a silicon substrate were successfully produced by using the polycarbonate membranes as templates. Because the polycarbonate template and surfactants were burned out simultaneously, the mesoporous rods were fabricated in one pot, which facilitates the production of the arrays in comparison with the previous work using PAA membranes [1]. In the case of mesoporous titania rods, the crystallization also occurred during calcination procedure. This facial synthesis method can be utilized to fabricate various types of metal oxide rods on a substrate.

\section{References}

[1] W. Zhu, Y. Han, and L. An, "Synthesis of ordered mesostructured silica nanotubal arrays," Microporous and Mesoporous Materials, vol. 84, no. 1-3, pp. 69-74, 2005.

[2] Z. Liang and A. S. Susha, "Mesostructured silica tubes and rods by templating porous membranes," Chemistry-A European Journal, vol. 10, no. 19, pp. 4910-4914, 2004.

[3] P. Lai, M. Z. Hu, D. Shi, and D. Blom, "STEM characterization on silica nanowires with new mesopore structures by spaceconfined self-assembly within nano-scale channels," Chemical Communications, no. 11, pp. 1338-1340, 2008.

[4] Y. Yamauchi, N. Suzuki, and T. Kimura, "Formation of mesoporous oxide fibers in polycarbonate confined spaces," Chemical Communications, no. 38, pp. 5689-5691, 2009.

[5] Y. Yamauchi, M. Sawada, M. Komatsu, et al., "Magnetically induced orientation of mesochannels in mesoporous silica films at 30 Tesla," Chemistry - An Asian Journal, vol. 2, no. 12, pp. 1505-1512, 2007.

[6] C.-W. Wu, T. Ohsuna, M. Kuwabara, and K. Kuroda, "Formation of highly ordered mesoporous titania films consisting of crystalline nanopillars with inverse mesospace by structural transformation," Journal of the American Chemical Society, vol. 128, no. 14, pp. 4544-4545, 2006.

[7] Y. Wu, G. Cheng, K. Katsov, et al., "Composite mesostructures by nano-confinement," Nature Materials, vol. 3, no. 11, pp. 816-822, 2004.

[8] D. Wang, R. Kou, Z. Yang, J. He, Z. Yang, and Y. Lu, "Hierachical mesoporous silica wires by confined assembly," Chemical Communications, no. 2, pp. 166-167, 2005.

[9] B. Platschek, N. Petkov, and T. Bein, "Tuning the structure and orientation of hexagonally ordered mesoporous channels in anodic alumina membrane hosts: a 2D small-angle X-ray scattering study," Angewandte Chemie-International Edition, vol. 45, no. 7, pp. 1134-1138, 2006.

[10] Y. Yamauchi, A. Sugiyama, M. Sawada, et al., "Magnetically induced orientation of mesochannels inside porous anodic alumina membranes under ultra high magnetic field of $30 \mathrm{~T}$ : confirmation by TEM," Journal of the Ceramic Society of Japan, vol. 116, no. 1359, pp. 1244-1248, 2008. 

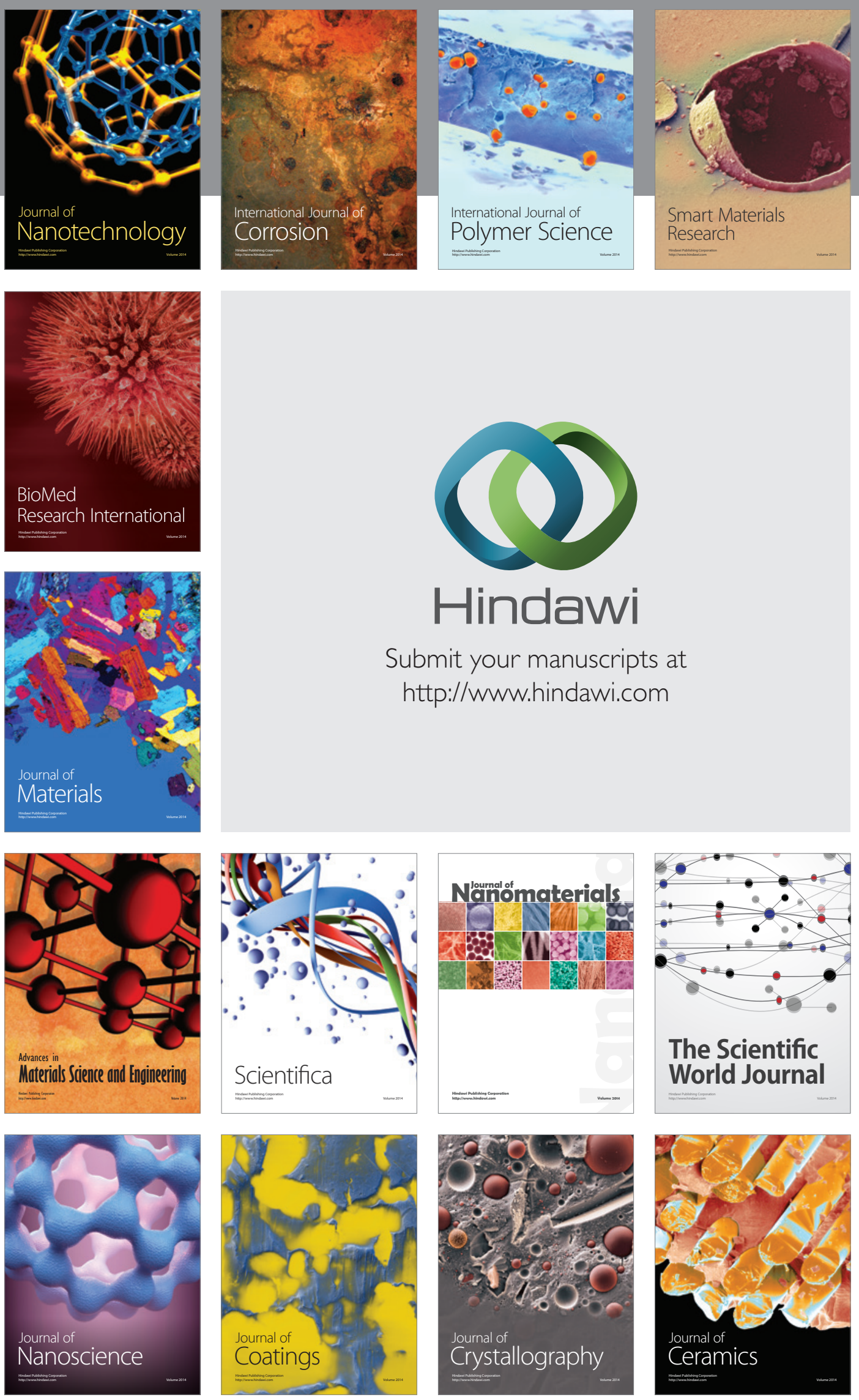

The Scientific World Journal

Submit your manuscripts at

http://www.hindawi.com

\section{World Journal}

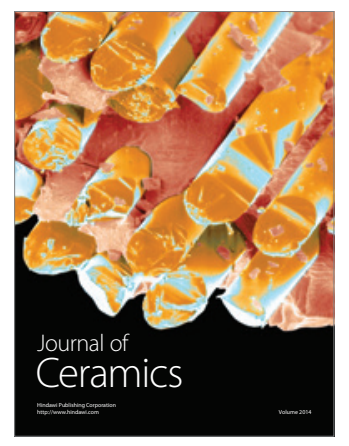

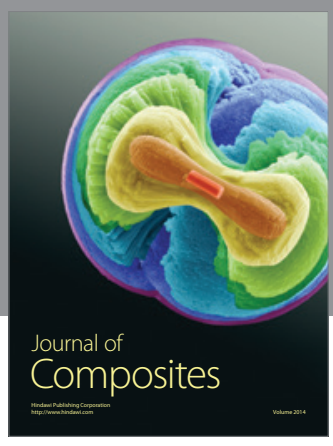
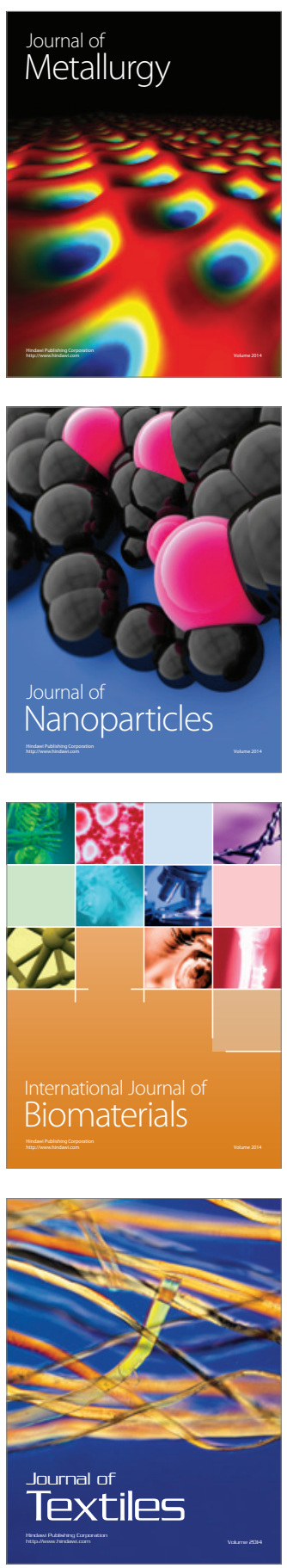